\title{
Vom allgemeinen Defizitmodell zum situationsspezifischen Rückfallrisiko - Anmerkungen zur Sucht-Neurose-Debatte
}

\author{
J. Lindenmeyer \\ salus klinik Lindow, Deutschland
}

Der Beitrag von Hand [2004] nimmt Bezug auf die vor einigen Jahren heftig geführte Debatte, ob pathologisches Glücksspiel eher als eine Suchtform oder als so genannte neurotische Störung verstanden bzw. behandelt werden sollte. Die damalige Polarisierung kann zwischenzeitlich als in vieler Hinsicht überwunden gelten. Stattdessen erlaubt der heutige Stand der Psychotherapieforschung eine wesentlich differenziertere Bestimmung sowohl der Gemeinsamkeiten als auch der Unterschiede beider Behandlungsparadigmen, die für eine indikationsgeleitete Behandlung von Glücksspielern relevant sein können.

Entgegen der Darstellung von Hand besteht hinsichtlich der Annahmen über die Entstehung und insbesondere die Funktionalität des Problemverhaltens kein Unterschied mehr zwischen dem «Neurosemodell» und aktuellen Suchtmodellen. Auch süchtiges Verhalten wird als gelerntes Verhalten betrachtet, das über positive und negative Verstärkung zu einer immer einseitigeren Bewältigungsform für persönliche Defizite werden kann, auf das zu verzichten sich der Betroffenen schließlich nicht mehr vorstellen kann. Entsprechend erfolgt die Suchtbehandlung hierzulande nicht in der von Hand skizzierten Art und Weise. Vielmehr zeichnen sich insbesondere stationäre Suchtbehandlungseinrichtungen durch ein umfangreiches interdisziplinäres Behandlungsangebot aus. Aus diesem wird vor dem Hintergrund einer sorgfältigen Indikationsstellung ein individueller Therapieplan zusammengestellt. Ebenso wie in der von Hand und seinen Mitarbeitern entwickelten multimodalen Verhaltenstherapie bei Spielern [Klepsch et al., 1989] erfolgt hierbei die Behandlung von Suchtkranken nach dem Kompensationsparadigma, um den Betroffenen den Verzicht auf ihr Problemverhalten durch den Aufbau von alternativen psychischen und sozialen Ressourcen zu ermöglichen.

Der tatsächliche Unterschied zwischen aktuellen Suchtmodellen und dem von Hand [2004] vertretenen «Neurosemodell» liegt vielmehr einzig in der angenommenen Automatisierung bzw. neurobiologischen Verankerung des symptomatischen Verhaltens. Für Hand bleibt über die gesamte Störungsentwicklung der funktionale Zusammenhang des Glückspielens als Vermeidungsverhalten/Ersatzlösung entscheidend. Entsprechend konzentriert sich die multimodale Verhaltenstherapie auf alternative Bewältigungsstrategien der auslösenden Konfliktsituationen, das Spielverhalten wird dadurch an Bedeutung verlieren und bei gelungener Therapie sogar vollkommen überflüssig. Psychophysiologische und neurobiologische Studien zur Sucht deuten dagegen darauf hin, dass der tatsächlich erlebten Verstärkung im Verlauf einer Suchtentwicklung immer geringere Bedeutung zukommt, während das Belohnungssystem im menschlichen Gehirn immer empfänglicher auf jene situativen Trigger reagiert, die eine früher erlebte positive Suchmittelwirkung ankündigen, unabhängig davon, ob diese aktuell noch eintritt oder nicht. Die Ergebnisse zu den in diesem Zusammenhang gegenwärtig am intensivsten empirisch untersuchten Paradigmen [vgl. Grüsser und Wölfling 2003; Lindenmeyer, 2001] stimmen darin überein, dass es sich hierbei um eine durch Lernen entstandene, dauerhafte neurostrukturelle Veränderung des Gehirns handelt, weswegen auch bildlich von der Entwicklung eines so genannten «Suchtgedächtnisses» gesprochen wird, das auch nach erfolgreichem Entzug oder längerer Abstinenz durch situative Trigger angesprochen werden kann. Ähnliche Phänomene wurden mittlerweile auch für andere psychische Störungen festgestellt, weswegen man ja auch von einem «Angstgedächtnis», einem «Schmerzgedächtnis» bzw. spezifischen, situativ getriggerten Informationsverarbeitungsauffälligkeiten bei Zwangspatienten oder depressiven Patienten spricht. In all diesen Fällen kann sich die Behandlung nicht darauf beschränken, den Betroffenen den Verzicht auf die Verstärkungswirkung ihres Problemverhaltens durch die Bereitstellung von Alternativressourcen zu erleichtern, sondern sie

\begin{tabular}{ll}
\hline KARGER & @ 2004 S. Karger GmbH, Freiburg \\
Fax +497614520714 & Accessible online at: \\
$\begin{array}{l}\text { E-mail Information@Karger.de } \\
\text { www.karger.com }\end{array}$ & www.karger.com/ver
\end{tabular}


muss in Form einer gezielten Rückfallprävention die Betroffenen zusätzlich dazu befähigen, in persönlich relevanten Rückfallrisikosituationen, die automatisch reaktivierte Tendenz zu ihrem Problemverhalten zu überwinden bzw. unter Kontrolle zu halten.

Spezifisch für die Situation von stoffgebundenen Süchten ist lediglich, dass die Einnahme eines Suchtmittels unmittelbar die rationalen Entscheidungs-, Bewertungs- und Steuerungsprozesse des Betroffenen beeinträchtigt. Außerdem können bei länger anhaltendem Substanzmissbrauch chronische kognitive Defizite und Beeinträchtigungen entstehen. Die Folge beider Beeinträchtigungen ist, dass den durch Sensitivierungsbzw. Gedächtniseffekte subkortikal verstärkten Anreizprozessen auf suchtspezifische Stimuli nunmehr eine beeinträchtigte kortikale Kontrolle gegenübersteht. Bildlich gesprochen haben sich die Machtverhältnisse zwischen Großhirn und Zwischenhirn dauerhaft verschoben, was die Gefahr eines Rückfalls ebenso wie die Schwierigkeit, einen Rückfall wieder zu stoppen, besonders erhöht. Entsprechend hat die Entwicklung spezifischer therapeutischer Interventionen zur Rückfallprävention in der Suchtbehandlung ihren Ausgangspunkt gehabt, um dann allerdings mit erheblichem Abstand auch in die Verhaltenstherapie anderer psychischer Störungen Eingang zu finden. Entsprechend ähneln die jeweils innerhalb der verhaltenstherapeutischen Behandlung bei somatoformen Schmerzstörungen, Angsterkrankungen, Zwangserkrankungen und Essstörungen entwickelten Verfahren zum Symptommangement in vivo (z.B. Expositionsbehandlung, Workhardening, Körperschemaexposition) in vivo in ihrer situativen Rückfallpräventionsorientierung den Interventionen innerhalb der Suchtbehandlung.

Es ist daher zweitrangig, ob Glücksspieler nach dem Suchtmodell oder dem Neurosemodell behandelt werden, da die Unterschiede zwischen beiden Behandlungsansätzen sehr gering geworden sind. Dagegen erscheint es über die von Hand vorgeschlagene Differenzierung der Behandlung von Glücksspielern nach der im Einzelfall bestehenden Vorrangigkeit von positiver oder negativer Verstärkung hinaus dringend notwendig abzuklären, inwieweit die Betroffenen einer symptomorientierten Rückfallprophylaxe aufgrund einer neurobiologisch verankerten Automatisierung des Spielverhaltens in bestimmten Situationen bedürfen. Da es sich bei einer solchen Sensitivierung des Belohnungssystems auf störungsspezifische Stimuli um unterschwellige Wahrnehmungs-, Aufmerksamkeits- und Gedächtniseffekte handelt, haben die Betroffenen mitunter wenig Bewusstheit darüber, warum sie ihr Problemverhalten immer wieder ausführen. Sie können sich oft selbst nicht verstehen und sind insbesondere befremdet darüber, wenn sie nach längerer Abstinenz wieder rückfällig werden, obwohl sie die Schädlichkeit dieses Tuns längst erkannt haben. Entsprechend erwies sich eine Befragung der Betroffenen gar in Form von Fragebogen in der bisherigen Rückfallforschung als wenig valide. Wesentlich Erfolg versprechender erscheint hier die klinische Nutzung von psychophysiologischen Messungen in Verhaltensexperimenten und Untersuchungsparadigmen aus der Wahrnehmungs-, Informationsverarbeitungs- und Gedächtnispsychologie.

\section{Literatur}

Grüsser SM, Wölfling K: Drogenverlangen - ein integrativer psychophysiologischer Erklärungsansatz. Suchtmedizin 2003;5:167-170.

Hand I: Negative und positive Verstärkung bei pathologischem Glücksspielen: Ihre mögliche Bedeutung für Theorie und Therapie von Zwangsspektrumsstörungen. Verhaltenstherapie 2004;14:133-144.

Klepsch R, Hand I, Wlatzlo Z, Kaunisto E, Friedrich B: Pathologisches Spielen; in Hand I, Wittchen HU (Hrsg): Verhaltenstherapie in der Medizin. Heidelberg, Springer, 1989, pp 313-326.

Lindenmeyer J: Neue Erklärungsansätze zur Behandlung von Verlangen nach Alkohol; in Dohrenbusch R, Kaspers F (Hrsg): Fortschritte der Klinischen Psychologie und Verhaltensmedizin. Lengerich, Pabst Publishers, 2001, pp 147-162. 